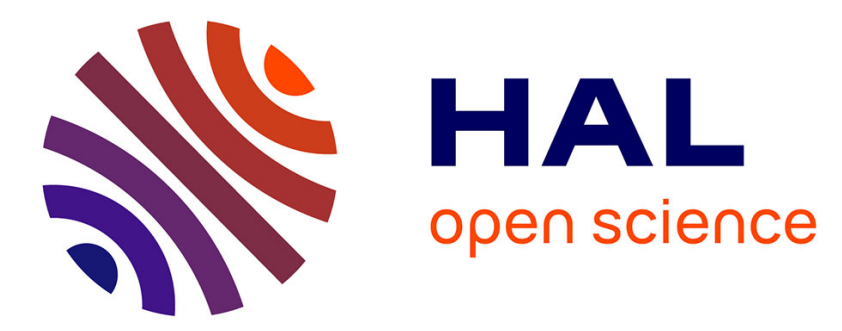

\title{
Hybrid systems with continuous-time inputs: subtleties in solution concepts and existence results
}

\author{
Maurice Heemels, Pauline Bernard, Koen J A Scheres, Romain Postoyan, \\ Ricardo G Sanfelice
}

\section{- To cite this version:}

Maurice Heemels, Pauline Bernard, Koen J A Scheres, Romain Postoyan, Ricardo G Sanfelice. Hybrid systems with continuous-time inputs: subtleties in solution concepts and existence results. 60th IEEE Conference on Decision and Control, CDC 2021, Dec 2021, Austin, United States. hal-03436863

\section{HAL Id: hal-03436863 https://hal.science/hal-03436863}

Submitted on 19 Nov 2021

HAL is a multi-disciplinary open access archive for the deposit and dissemination of scientific research documents, whether they are published or not. The documents may come from teaching and research institutions in France or abroad, or from public or private research centers.
L'archive ouverte pluridisciplinaire HAL, est destinée au dépôt et à la diffusion de documents scientifiques de niveau recherche, publiés ou non, émanant des établissements d'enseignement et de recherche français ou étrangers, des laboratoires publics ou privés. 


\title{
Hybrid Systems with Continuous-time Inputs: Subtleties in Solution Concepts and Existence Results
}

\author{
W.P.M.H. Heemels, P. Bernard, K.J.A. Scheres, R. Postoyan, R. G. Sanfelice
}

\begin{abstract}
We study solution concepts and their properties for hybrid systems that can flow and jump, affected by continuous-time inputs. While the solution concepts, the existence of solutions and (forward) completeness properties are extensively discussed in the absence of (external) inputs, there are surprisingly few results when inputs are present, certainly in the case where the flow and jump sets depend on the inputs. Given the relevance of this class of hybrid systems for many applications such as hybrid or networked control for plants subject to disturbances or measurement noise, we discuss in this paper notions of solutions in the presence of inputs and show through various examples the subtleties that can occur. Moreover, we provide tools to guarantee the existence of solutions and results to establish completeness properties.
\end{abstract}

\section{INTRODUCTION}

In this paper we are interested in the class of hybrid systems as advocated in, e.g., [1]-[3]. As such, we consider systems $\mathcal{H}=(C, F, D, G, W)$ of the form

$$
\begin{cases}\dot{x} \in F(x, w), & (x, w) \in C, \\ x^{+} \in G(x, w), & (x, w) \in D,\end{cases}
$$

where $x$ denotes the state taking values in $\mathbb{R}^{n_{x}}$, and $w$ the (disturbance) input taking values in $W \subseteq \mathbb{R}^{n_{w}}$. Moreover, $C \subseteq \mathbb{R}^{n_{x}} \times \mathbb{R}^{n_{w}}$ is the flow set, $D \subseteq \mathbb{R}^{n_{x}} \times \mathbb{R}^{n_{w}}$ the jump set, $F: \mathbb{R}^{n_{x}} \times \mathbb{R}^{n_{w}} \rightrightarrows \mathbb{R}^{n_{x}}$ the flow map and $G: \mathbb{R}^{n_{x}} \times$ $\mathbb{R}^{n_{w}} \rightrightarrows \mathbb{R}^{n_{x}}$ the jump map, where $F$ and $G$ are possibly setvalued. For general hybrid systems, the question of definition of solutions, as well as their existence and properties has been an intriguing and important matter. It received ample attention for various classes of systems, see, e.g., [4]-[6]. For the class of systems (1), these questions were also extensively discussed in [1] in the absence of external inputs, i.e., without $w$ in (1). Moreover, the existence of (nontrivial) solutions and completeness of so-called maximal solutions were studied in detail, see, in particular, [1, Prop. 2.10, Prop. 6.10].

Maurice Heemels and Koen Scheres are with the Control Systems Technology Section, Dept. Mechanical Engineering, Eindhoven University of Technology, The Netherlands. m.heemelsatue.nl, k.j.a.scheres@tue.nl

Pauline Bernard is with the Centre Automatique et Systèmes, MINES ParisTech, Université PSL, Paris, France. pauline.bernard@mines-paristech.fr.

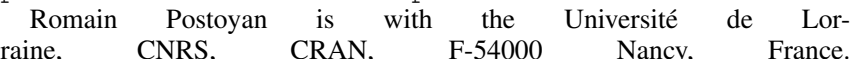
romain.postoyanduniv-lorraine.fr. His work is supported by the ANR under grant HANDY ANR-18-CE40-0010.

Ricardo G. Sanfelice is with the Department of Electrical and Computer Engineering, University of California, Santa Cruz, CA 95064, USA. ricardo@ucsC. edu. Research partially supported by the NSF under Grants no. ECS-1710621, CNS-1544396, and CNS-2039054, by AFOSR under Grants no. FA9550-19-1-0053, FA9550-19-1-0169, and FA9550-201-0238, and by ARO under Grant no. W911NF-20-1-0253.
Works on hybrid systems of the type (1) with inputs are available in the literature, considering the case where either

I) combinations of input and state trajectories (solution pairs) are sought and hence, the input is 'free', see, e.g., [2], [7]; or,

II) the input is 'given' a priori and we search for a state trajectory only [8].

To elaborate, in [2], [7] the input signals are considered as hybrid signals, meaning that they are defined on hybrid time domains involving both real time $t \in \mathbb{R}_{\geqslant 0}$ and jump counter $j \in \mathbb{N}$, see also Definition 1 below. Between jumps, the input is Lebesgue measurable and locally essentially bounded. In [2], [7], it is enforced that the domain of the input is the same as the domain of the solution itself. In certain cases this may be unnatural from a modelling perspective, as often the input is a priori given and the domain of the solution is a consequence of the hybrid dynamics and possibly the hybrid time domain of the input, but not a priori taken equal. In [7], a basic existence result of solution pairs is presented and the properties of maximal solutions (maximal in the sense that they cannot be prolonged) are characterised. Interestingly, as the domains of input and state have to be a priori the same, the existence result is, loosely speaking, stated as: "given an initial state, there is a combination of an input signal and a state signal (on the same hybrid time domain) that form together a solution pair". We are not aware of any result regarding the existence of solutions when the input signal is specified a priori.

In [8], perspective II) is taken in which a hybrid input is given a priori, however, without results regarding the existence of (nontrivial) solutions or an analysis of (forward) completeness properties of solutions. To be more precise, in [8] the hybrid time domains of inputs and states do not have to match, but the domain of the state is a consequence of that of the input, and, of course, the hybrid dynamics. Motivated by interconnections of hybrid systems, in [8] the inputs are considered to be hybrid arcs, which imposes that between jumps the input is locally absolutely continuous, and not just Lebesgue measurable as in [2], [7]. Another interesting difference between [2] on the one hand and [7], [8] on the other hand is that [7], [8] require the solution during flow phases to satisfy the flow constraint $(x, w) \in C$ at all times (except the beginning and the end of the flow - to be able to 'flow' from the boundary of $C$ into $C$ ), while in [2] the flow constraint $(x, w) \in C$ has only to be satisfied 'almost everywhere' (a.e.) during flow. Hence, in [7], [8] the set $C$ is seen as a hard constraint, which should 
always be satisfied except at the beginning and end of flows, while in [2] the constraint may be violated on a measure zero set during flow, which is sufficient in order to study and guarantee (input-to-state) stability properties using Lyapunov theory. Interestingly, under closure of $C$ and without inputs, both solutions reduce to the same concept, the one as used in [1], due to continuity of the state trajectory during flow phases.

Motivated by the gap in the literature regarding the study of existence and completeness properties of solutions to (1) given an input and the importance of this question in many application settings including hybrid control [9]-[12], networked control [13], [14] and event-triggered control [15][17] for plants subject to disturbances and/or measurement noise, we study in this paper the scenario where the input in (1) is fixed a priori and is a continuous-time measurable input defined on the nonnegative real line $\mathbb{R}_{\geqslant 0}$. Indeed, for these applications, it would be welcome to have such existence and completeness results, as they are often needed to guarantee that the designed controllers are well-posed in presence of external disturbance and noise signals. To fill this gap, we start by discussing definitions of a solution to $\mathcal{H}$ with continuous-time measurable inputs and indicate the subtleties and consequences of using each possible variant of solution concepts. Moreover, we provide tools to guarantee the existence of (nontrivial) solutions (given an initial state and an input signal) and their (forward) completeness properties. Herein we will differentiate between the variations in the solution concepts and the conditions imposed on the external input signal.

This paper is further organized as follows. After introducing some notational conventions, in Section II we discuss possible solution concepts for the case where the input $w$ is a continuous-time measurable function. In Section III we provide results on the existence and completeness of solutions given an input. In Section IV we show how less stringent conditions for existence and completeness of solutions can be obtained if the continuous-time input is of a piecewise continuous nature. Conclusions close the paper in Section V. Notation: The sets of all nonnegative and positive integers are denoted $\mathbb{N}$ and $\mathbb{N}_{>0}$, and the set of rational numbers by $\mathbb{Q}$. The sets of reals and nonnegative reals are indicated by $\mathbb{R}$ and $\mathbb{R}_{\geqslant 0}$. By $|\cdot|$ we denote the Euclidean norm. For $z \in \mathbb{R}^{n}$ and $r \geqslant 0$ we denote by $B(x, r)$ the closed ball of radius $r$ around $z$, i.e., $B(z, r)=\left\{x \in \mathbb{R}^{n}|| x-z \mid \leqslant r\right\}$. The interior of a set $A \subseteq \mathbb{R}^{n}$ is denoted by int $A$ and its closure by $\bar{A}$. We denote the complement $D^{c}$ of a set $D \subseteq \mathbb{R}^{n}$ as $D^{c}:=\left\{x \in \mathbb{R}^{n} \mid x \notin D\right\}$.

\section{SOLUTION CONCEPT FOR MEASURABLE INPUTS}

To formally introduce the solution concept, we need the following definitions, which are taken from [1], [2], [7].

Definition 1. A subset $E \subset \mathbb{R}_{\geqslant 0} \times \mathbb{N}$ is a compact hybrid time domain, if $E=\bigcup_{j=0}^{J-1}\left(\left[t_{j}, t_{j+1}\right], j\right)$ for some finite sequence of times $0=t_{0} \leqslant t_{1} \leqslant t_{2} \leqslant \ldots \leqslant t_{J}$. It is a hybrid time domain, if for all $(T, J) \in E, E \cap([0, T] \times 0,1, \ldots, J)$ is a compact hybrid time domain. A hybrid signal is a function defined on a hybrid time domain.

For a hybrid time domain $E, \sup _{t} E$ := $\sup \left\{t \in \mathbb{R}_{\geqslant 0}: \exists j \in \mathbb{N}\right.$ such that $\left.(t, j) \in E\right\}, \quad \sup _{j} E \quad:=$ $\sup \left\{j \in \mathbb{N}: \exists t \in \mathbb{R}_{\geqslant 0}\right.$ such that $\left.(t, j) \in E\right\}$ and $\sup E:=$ $\left(\sup _{t} E, \sup _{j} E\right)$.

Definition 2. A hybrid signal $\phi$ is called a hybrid arc, if $\phi(\cdot, j)$ is locally absolutely continuous for each $j$.

Definition 3. [1], [2] For $W \subseteq \mathbb{R}^{n_{w}}$ and $n_{w} \in \mathbb{N}_{>0}$, we define the class of inputs $\mathcal{L}_{W}$ as all functions $w: \mathbb{R}_{\geqslant 0} \rightarrow$ $W$ that are Lebesgue measurable and locally essentially bounded.

As stated in the introduction, we consider inputs that are defined on $\mathbb{R}_{\geqslant 0}$, which may model disturbance signals or measurement noise acting on hybrid, networked, and eventtriggered controlled systems. Throughout this paper, we have the following standing assumption.

Standing Assumption. The sets $C$ and $W$ are closed.

Inspired by [2], [7], we introduce two solution concepts.

Definition 4. A hybrid arc $\phi$ is an e-solution to $\mathcal{H}$ for input $w \in \mathcal{L}_{W}$, if

(S1-e) for all $j \in \mathbb{N}$ such that $I^{j}:=\{t:(t, j) \in \operatorname{dom} \phi\}$ has nonempty interior, it holds that $\dot{\phi}(t, j) \in$ $F(\phi(t, j), w(t))$ for almost all $t \in \operatorname{int} I^{j}$ and $(\phi(t, j), w(t)) \in C$ for all $t \in \operatorname{int} I^{j}$;

(S2) for all $(t, j) \in \operatorname{dom} \phi$ such that $(t, j+1) \in$ $\operatorname{dom} \phi,(\phi(t, j), w(t)) \in D$ and $\phi(t, j+1) \in$ $G(\phi(t, j), w(t))$.

It is an ae-solution for input $w$, if (S2) holds together with

(S1-ae) for all $j \in \mathbb{N}$ such that $I^{j}:=\{t:(t, j) \in \operatorname{dom} \phi\}$ has nonempty interior, $\dot{\phi}(t, j) \in F(\phi(t, j), w(t))$ and $(\phi(t, j), w(t)) \in C$ hold for almost all $t \in I^{j}$.

A few comments are in order. First of all, observe that an e-solution is also an ae-solution. Secondly, note that the ae-solution concept is in line with [2, Section 2], where the flow constraint is satisfied almost everywhere during flow intervals - though, as a difference to [2, Section 2], an aesolution is here defined for a given continuous-time input $w$. In contrast, in e-solutions, the flow constraint $(x, w) \in C$ has to hold "everywhere" in the interior of flow intervals (but not necessarily at the boundaries of the flow interval). The e-solutions are closer in nature to [7], [8] (although note that in [2], [3], [7], [8] hybrid inputs on hybrid time domains are used instead of continuous-time functions defined on $\mathbb{R}_{\geqslant 0}$ ). Thirdly, in [8], (S1-e) was used in the notion of solution, but given the fact that during flow the input is required to be locally absolutely continuous, (S1-e) and (S1-ae) coincide in their setting. Moreover, generally we can state that if the input $w$ is continuous, then both solution concepts coincide as $C$ is closed (standing assumption). In fact, in the special case of no inputs, ae-solutions are e-solutions due to local absolute continuity of hybrid arcs during flow intervals, and we recover the solution concept in [1]. However, in a general 
setting where the input $w$ models a possibly discontinuous noise/disturbance, both definitions do not coincide as will be illustrated below. One may choose to use one or the other depending on the context and the intended purposes. In any case, we are interested in this paper to study the existence and completeness properties for both e-solutions and ae-solutions given an input. We will start doing this in the next section, after providing some useful terminology and two examples.

Definition 5. An (e or ae-)solution $\phi$ to $\mathcal{H}$ for a given input $w \in \mathcal{L}_{W}$ is called nontrivial, if $\operatorname{dom} \phi$ contains at least two points. It is said to be maximal, if there does not exist another solution $\psi$ to $\mathcal{H}$ for the same input $w$ such that $\operatorname{dom} \phi$ is a proper subset of $\operatorname{dom} \psi$ and $\phi(t, j)=\psi(t, j)$ for all $(t, j) \in \operatorname{dom} \phi$. We denote the set of all maximal $e$ solutions and ae-solutions to $\mathcal{H}$ for input $w$ by $\mathcal{S}_{\mathcal{H}}^{e}(w)$ and $\mathcal{S}_{\mathcal{H}}^{a e}(w)$, respectively. We say that the solution is complete, if dom $\phi$ is unbounded, and we say that it is $t$-complete, if $\sup _{t} \operatorname{dom} \phi=\infty$.

The next two examples show how changes to inputs on a set of measure zero of their domain can affect the corresponding solutions thereby also highlighting subtle differences between e- and ae-solutions. Moreover, the examples also demonstrate subtleties and problems regarding the existence of nontrivial solutions and the completeness of maximal solutions.

Example 1 (Activating jumps by measure zero changes to inputs). Consider the hybrid model

$$
\dot{x}=0 \quad x+w \geqslant 0, \quad x^{+}=x+1 \quad x+w \leqslant 0,
$$

where we take three different input signals $w_{i}, i \in\{1,2,3\}$ :

$$
\begin{aligned}
& w_{1}(t):=1, t \in \mathbb{R}_{\geqslant 0} \\
& w_{2}(t):= \begin{cases}1, & t \in \mathbb{R}_{\geqslant 0} \backslash\{1\}, \\
-1.5, & t=1 .\end{cases} \\
& w_{3}(t):= \begin{cases}1, & t \in\left(\mathbb{R}_{\geqslant 0} \backslash \mathbb{Q}\right) \cup\{0\}, \\
-1.5, & t \in \mathbb{Q} \backslash\{0\} .\end{cases}
\end{aligned}
$$

Here, the signals $w_{i}, i \in\{1,2,3\}$ are essentially equivalent in the " $\mathcal{L}_{\infty}$ " sense, i.e., $\left\|w_{i}-w_{j}\right\|_{\infty}=0$ for all $i, j \in$ $\{1,2,3\}$, however, the solutions corresponding to these inputs can be significantly different. Let us consider the initial state $\phi_{1}(0,0)=\xi=0$. For $w_{1}$, there is a unique maximal ae-solution $\phi_{1}(t, j)=0$ on domain $\operatorname{dom} \phi_{1}=\mathbb{R}_{\geqslant 0} \times\{0\}$, without any jumps, which is also an e-solution.

For input $w_{2}$, we obtain three maximal ae-solutions $\phi_{2}^{\ell}$, $\ell=0,1,2$ ( $\ell$ indicating the number of jumps at time $t=1$ ):

$$
\begin{gathered}
\phi_{2}^{0}(t, j)=0, \\
\phi_{2}^{1}(t, j)= \begin{cases}0, & (t, j) \in[0, \infty) \times\{0\} \\
1, & (t, j) \in[1, \infty) \times\{1\}\end{cases} \\
\phi_{2}^{2}(t, j)= \begin{cases}0, & (t, j) \in[0,1] \times\{0\}, \\
1, & (t, j)=\{1\} \times\{1\} \\
2, & (t, j) \in[1, \infty) \times\{2\} .\end{cases}
\end{gathered}
$$

Note that $\phi_{2}^{0}$ is not an e-solution, but $\phi_{2}^{1}$ and $\phi_{2}^{2}$ are.

Finally, for $w_{3}$ and again the same initial state $\xi=0$, we have that $\phi_{1}$ and $\phi_{2}^{\ell}, \ell=0,1,2$ are also ae-solutions, but there are more solutions as jumps may occur at any rational time. Hence, there are infinitely many ae-solutions. Since only two jumps are possible due to the data of (2), all those maximal solutions will be t-complete. On the other hand, observe that for $w_{3}$, system (2) does not admit a nontrivial e-solution.

In the above example, a measure zero change in the input had the effect of activating jump possibilities. On the other hand, the following example shows how measure zero change to the input signal can instead disable jumps and thereby prohibit the existence of a complete solution [1, Chapter 4].

Example 2 (Disabling jumps by measure zero changes to inputs). Consider the system

$$
\dot{x}=1, \quad x+w \in[0,1], \quad x^{+}=0, \quad x+w \in[1,2]
$$

and take $\phi(0,0)=0$, and $w(t)=0, t \in \mathbb{R}_{\geqslant 0}$, which leads to a sawtooth ae-and e-solution that is t-complete. However, if we apply a zero measure change to this input and have $w(t)=0$ for $t \neq 1$ and $w(1)=3$, then the jump is 'disabled' at $t=1$, causing a deadlock in the sense that the maximal ae-and e-solution has domain $[0,1] \times\{0\}$. Hence, in this case the set of all solutions is smaller than with the original input.

The above examples motivate the development of tools to guarantee existence and completeness of e- and ae-solutions.

\section{EXISTENCE AND COMPLETENESS OF SOLUTIONS}

In this section we start by providing conditions for the existence of nontrivial solutions and their properties for hybrid systems of the form (1), thereby extending the results in [1, Proposition 2.10] without (external) inputs and in [7, Proposition 3.4] for a hybrid input to the case of continuoustime measurable inputs. After that we provide examples illustrating the results.

The following set will play an important role below:

$$
C_{0}:=\left\{\xi_{0} \in \mathbb{R}^{n_{x}} \mid \exists w_{0} \in W \text { s.t. }\left(\xi_{0}, w_{0}\right) \in C\right\} .
$$

Indeed, if a point $\xi \notin C_{0}$ and $(\xi, w(0)) \notin D$, then the set of (nontrivial) solutions $\phi$ with $\phi(0,0)=\xi$ and input $w$ is empty, see also Lemma 7 below.

Proposition 6. Consider the hybrid system $\mathcal{H}=$ $(C, F, D, G, W)$.

(i) There exists a nontrivial e-solution $\phi$ to $\mathcal{H}$ for input $w \in$ $\mathcal{L}_{W}$ with $\phi(0,0)=\xi \in \mathbb{R}^{n_{x}}$ if and only if $(\xi, w(0)) \in$ $D$ or

(VC-e): there exist $\epsilon>0$ and an absolutely continuous function $z:[0, \epsilon] \rightarrow \mathbb{R}^{n_{x}}$ such that $z(0)=\xi, \dot{z}(t) \in F(z(t), w(t))$ for almost all $t \in[0, \epsilon]$ and $(z(t), w(t)) \in C$ for all $t \in(0, \epsilon)$.

(ii) If condition (VC-e) holds for all $\xi \in C_{0}$ and all $w \in$ $\mathcal{L}_{W}$ with $(\xi, w(0)) \notin D$, then for all $\bar{w} \in \mathcal{L}_{W}$ every 
maximal e-solution $\phi \in \mathcal{S}_{\mathcal{H}}^{e}(\bar{w})$ satisfies exactly one of the following properties:

(a) $\phi$ is complete;

(b) $\phi$ is not complete and 'ends with flow': $\operatorname{dom} \phi$ is bounded and the interval $I^{J}:=\{t:(t, J) \in \operatorname{dom} \phi\}$ with $J=\sup _{j} \operatorname{dom} \phi$ is open to the right, and there does not exist an absolutely continuous function $z: \overline{I^{J}} \rightarrow$ $\mathbb{R}^{n_{x}}$ satisfying $\dot{z}(t) \in F(z(t), \bar{w}(t))$ for almost all $t \in I^{J}$ and $(z(t), \bar{w}(t)) \in C$ for all $t \in \operatorname{int} I^{J}$, and such that $z(t)=\phi(t, J)$ for all $t \in I^{J}$;

(c) $\phi$ is not complete and 'ends with a jump': $\operatorname{dom} \phi$ is bounded with $(T, J):=\sup \operatorname{dom} \phi \in \operatorname{dom} \phi, I^{J}=\{T\}$, $(\phi(T, J), \bar{w}(T)) \notin D$ and $\phi(T, J) \notin C_{0}$.

When (VC-e) is replaced by

(VC-ae): there exist $\epsilon>0$ and an absolutely continuous function $z:[0, \epsilon] \rightarrow \mathbb{R}^{n_{x}}$ such that $z(0)=\xi$, $\dot{z}(t) \in F(z(t), w(t))$ and $(z(t), w(t)) \in C$ for almost all $t \in[0, \epsilon]$,

then all the above statements apply to ae-solutions, where in (b) the phrase " $(z(t), \bar{w}(t)) \in C$ for all $t \in I^{J}$ " is replaced by " $(z(t), \bar{w}(t)) \in C$ for almost all $t \in I^{J}$.”

An important observation is that (VC-ae) and (VC-e) are independent of the value of $w(0)$. We will see some consequences of this fact in the examples below.

Note that for the hypothesis in (ii), namely ( $\mathrm{VC}-\mathrm{e}$ ) (or (VC-ae)) to hold for all $\xi \in C_{0}$ and $w \in \mathcal{L}_{W}$ with $(\xi, w(0)) \notin D$, it is equivalent to require that (VC-e) (resp., (VC-ae)) holds for all $\xi \in \widetilde{C}_{0}$, and all $w \in \mathcal{L}_{W}$, where

$$
\widetilde{C}_{0}=\left\{\xi \in C_{0} \mid \exists w^{\prime} \in W \text { s.t. }\left(\xi, w^{\prime}\right) \notin D\right\},
$$

since $w \in \mathcal{L}_{W}$ implies also that $\widetilde{w}$ defined by $\widetilde{w}(0)=w^{\prime}$ and $\widetilde{w}(t)=w(t), t>0$, satisfies $\widetilde{w} \in \mathcal{L}_{W}$. In fact, generally we can replace "for every $\xi \in C_{0}$ and $w \in \mathcal{L}_{W}$ with $(\xi, w(0)) \notin$ $D$ " by "for every $\xi \in \widetilde{C}_{0}$ and any $w \in \mathcal{L}_{W}$."

Note also that having existence and completeness of an e-solution for a given input does not mean much when the input is changed, even if the change is at an isolated time in the interior of a flow interval.

Next, let us explain the role of $C_{0}$ a bit more via the following property enabled by the standing assumption.

Lemma 7. Let an absolutely continuous function $z, w \in \mathcal{L}_{W}$ and $\epsilon>0$ be such that (VC-ae) holds for some $\xi \in \mathbb{R}^{n_{x}}$. Then, $z(t) \in C_{0}$ for all $t \in[0, \epsilon]$.

The interest of this lemma is that it shows that for (VC-ae) or (VC-e) to hold, we have to consider an initial state in $C_{0}$, and that during flow the state will not leave $C_{0}$. So, even a discontinuity in $w$ at $t=\epsilon$ cannot get the state outside $C_{0}$.

Let us illustrate the use of Proposition 6 in an example.

Example 3. Consider a controlled system in which the state $i$ s aimed to be kept inside the set $[-1.7,1.7]$ by resetting it based on noisy measurements. The measurement noise satisfies $w(t) \in W:=[-0.2,0.2]$ for any $t \in \mathbb{R}_{\geqslant 0}$ and the controlled system is given by

$$
\begin{cases}\dot{x}=x & |x+w| \leqslant 1.5, \\ x^{+} \in[-0.1,0.1] & |x+w| \geqslant 1 .\end{cases}
$$

To apply Proposition 6, note that $C_{0}=[-1.7,1.7]$. In case $\xi \in C_{0}$ and $w \in W$ with $(\xi, w(0)) \notin D$, the latter implies that $|\xi|<1.2$ as $|\xi+w(0)|<1$ and $|w(0)| \leqslant 0.2$. Given the definition of the flow set $C=\{(x, w)|| x+w \mid \leqslant 1.5\}$, we see that as long as $|x| \leqslant 1.3$ the inequality $|x+w| \leqslant 1.5$ is guaranteed. This shows that (VC-e) and (VC-ae) are both satisfied as it takes some positive time for the solution to $\dot{x}=x$ to grow from an absolute value below 1.2 to an absolute value larger or equal than 1.3. Hence, the existence of nontrivial ae- and e-solutions is guaranteed and any maximal solution satisfies only one of the properties (a)-(c) in Proposition 6. In fact, it can be deduced that only (a) can hold and thus all maximal (e-or ae-) solutions to $\mathcal{H}$ for input $w$ are complete - in fact, t-complete. This property holds for any $w \in \mathcal{L}_{W}$.

Note that for any $w \in \mathcal{L}_{W}$, each (e-and ae-) solution $\phi$ remains indeed in $[-1.7,1.7]$, so forward invariance of this set is guaranteed despite the presence of measurement noise.

There are some subtleties with Proposition 6 due to the use of merely measurable inputs, as will be demonstrated with the next reworked example, where the overlap between $C$ and $D$ is smaller than in Example 3.

Example 4. We modify Example 3 by restricting the flow set to obtain

$$
\begin{cases}\dot{x}=x & |x+w| \leqslant 1 \\ x^{+} \in[-0.1,0.1] & |x+w| \geqslant 1\end{cases}
$$

and still adopt $W:=[-0.2,0.2]$. In this case, for $\phi(0,0)=$ $\xi=1$ and $w(t)=-0.2$ if $t=0$ and $w(t)=0.2$ if $t>0$, no jump and no flow are possible: a jump is not possible as the jump condition $|\phi(0,0)+w(0)| \geqslant 1$ is not satisfied and both (VC-ae) and (VC-e) are not satisfied, as, loosely speaking, $|x+w| \leqslant 1$ cannot be satisfied on a nontrivial flow interval. Essentially, the problematic issue for existence of nontrivial solutions is that the possibility of a jump at $t=0$ depends on $w(0)$ (i.e, $(\xi, w(0)) \in D$ is needed) and the value of $w(0)$ does not affect the satisfaction of $(V C$-ae) or $(V C-e)$. Informally, the information $(\xi, w(0)) \notin D$ does not restrict $\xi$ sufficiently (as in Example 3) to conclude that flow is possible for $w(t), t>0$.

Similar problems arise for

$$
\begin{cases}\dot{x}=x & |x+w| \leqslant c, \\ x^{+} \in[-0.1,0.1] & |x+w| \geqslant 1 .\end{cases}
$$

as long as $c<1.4$. Indeed, take $c=1.4-\eta$ for small positive $\eta$ and consider $\phi(0,0)=1.2-\frac{\eta}{2}$ and $w$ as above. No flow is possible ((VC-e) and (VC-ae) are both not satisfied) and a jump is not possible either.

In fact, if there are no restrictions on $w$, i.e., $W=\mathbb{R}$ in the example above, then $C_{0}=\mathbb{R}$ and (VC-ae) or (VC-e) 
have to hold for all $\xi \in \mathbb{R} \backslash D$, which is not satisfied for this example and typically also for many other systems. So, a tighter choice of $W$, namely precise information about the range of the inputs (or their regularity, see Section IV), is typically needed to guarantee the existence of nontrivial eor ae-solutions for all $w \in \mathcal{L}_{W}$. Otherwise, there is often not much one can guarantee.

\section{RESTRICTING THE INPUT SPACE TO CÀDLÀG FUNCTIONS}

As we have seen in Example 4 and as illustrated on another example below, the existence of nontrivial solutions for all Lebesgue measurable inputs, taking values in $W$, is often hard to guarantee apart from rather particular cases, such as Example 3, where the bounds on $w$ and the 'margin' between $C$ and $D$ allow to guarantee existence of nontrivial solutions for all $w \in \mathcal{L}_{W}$. We will show in this section that under an additional regularity assumption on the input, namely that it is càdlàg (see Definition 8 below), the existence of nontrivial solutions can be more easily guaranteed.

Example 5. Consider the system

$$
\dot{x}=0 \quad x+w \geqslant 0, \quad x^{+}=0 \quad x+w \leqslant 0
$$

with $x \in \mathbb{R}$ and $W=\mathbb{R}$. Interestingly, $C \cup D=\mathbb{R}^{2}$ and both $C$ and $D$ are closed. However, maybe counter-intuitively, it is not the case that nontrivial (e-or ae-)solutions exist for all initial states and Lebesgue measurable input functions. Let us take a closer look. Clearly, $C_{0}=\mathbb{R}$. Take $\phi(0,0)=\xi=1$, and select an input function with $w(0)$ such that $\xi+w(0)>0$ implying that a jump is not possible at the initial time. Moreover, take $w(t)=-2$ on $(0, \infty)$. For the existence of a nontrivial ae-solution, (VC-ae) has to be satisfied, which, unfortunately, is not the case for the given input. In fact, the class of Lebesgue measurable inputs is too large here to guarantee existence of nontrivial solutions for all states in $C_{0}$ and all inputs in $\mathcal{L}_{W}$, since (VC-ae) cannot always be satisfied (in fact, it has to be satisfied for all $\xi \in \widetilde{C}_{0}=\mathbb{R}$ and all $w \in \mathcal{L}_{W}$ in this case). This issue is actually related to the fact that $w(0)$ is totally 'decoupled' from $w(t), t>0$. As we will see in Example 6, if we impose a piecewise continuity property (càdlàg) on the inputs, instead of measurability only, the existence of nontrivial solutions in (12) can be guaranteed for all considered inputs.

The issues in the above example are due to the use of the very rich class of Lebesgue measurable inputs for which the value of $w(0)$ (important for jumps) is not related in any way to the function values $w(t)$ for times $t>0$ (important for flow, i.e., for the satisfaction of (VC-ae) or (VC-e)). In particular, the fact that a jump is not possible at $(\phi(0,0), w(0))$ normally gives information, i.e., $(\phi(0,0), w(0)) \notin D$, which forms a restriction on the initial conditions for which (VCae) or (VC-e) has to hold (in order to obtain existence results). Indeed, (VC-ae) or (VC-e) would be needed for all $(\phi(0,0), w(0)) \in \bar{C} \backslash D$ and thus $\phi(0,0) \in \widetilde{C}_{0}$ to obtain existence of nontrivial solutions, which relates to sets that are not 'small enough'. Only in examples such as Example 3, in which sufficient "margin exists between $D^{c}$ and int $C$ ", do the existence and completeness results apply for all $w \in$ $\mathcal{L}_{W}$ and all $\xi \in C_{0}$. However, the required conditions for existence and completeness of (maximal) solutions change significantly when we consider càdlàg inputs, which is still a rather rich class of inputs.

Definition 8. A function $w: \mathbb{R}_{\geqslant 0} \rightarrow \mathbb{R}^{n_{w}}$ is said to be càdlàg ("continue à droite, limite à gauche"), denoted by $w \in \mathcal{P C}$, when there exists a sequence $\left\{t_{i}\right\}_{i \in \mathbb{N}}$ with $t_{i+1}>$ $t_{i}>t_{0}=0$ for all $i \in \mathbb{N}$ and $t_{i} \rightarrow \infty$ when $i \rightarrow \infty$ such that $w$ is continuous on $\left(t_{i}, t_{i+1}\right)$ where $\lim _{t \uparrow t_{i}} w(t)$ exists for all $i \in \mathbb{N}_{>0}$ and $\lim _{t \downarrow t_{i}} w(t)$ exists for all $i \in \mathbb{N}$ with $\lim _{t \downarrow t_{i}} w(t)=w\left(t_{i}\right)$, i.e., $w$ is piecewise continuous, right continuous and left limits exist for each $t_{i}, i \in \mathbb{N}_{>0}$. Given a set $W \subseteq \mathbb{R}^{n_{w}}$, then we denote by $\mathcal{P} \mathcal{C}_{W}$ the set of functions $\left\{w \in \mathcal{P C} \mid w(t) \in W\right.$ for all $\left.t \in \mathbb{R}_{\geqslant 0}\right\}$.

Note that continuous functions are contained in $\mathcal{P C}$ as $\left\{t_{i}\right\}_{i \in \mathbb{N}}$ can then be chosen arbitrarily. In the next proposition we provide conditions for the existence of nontrivial solutions for hybrid system (1), with the $\mathcal{P C}$ restriction on inputs. Interestingly, note that checking ( $\mathrm{VC}$-ae) or (VC-e) for a $w \in \mathcal{P C}$, is equivalent to checking it for a continuous input as the restriction of $w$ to a small enough interval is continuous. Hence, since we consider closed sets $C$, this causes (VC-e) and (VC-ae) to coincide, and, therefore, we will have only one viability condition, (VC). Similarly, we show that in this context, e- and ae-solutions actually coincide.

Proposition 9. Consider the hybrid system $\mathcal{H}=$ $(C, F, D, G, W)$.

(i) Any ae-solution to $\mathcal{H}$ with input $w \in \mathcal{P} \mathcal{C}_{W}$ is also an e-solution, hereafter called "solution".

(ii) There exists a nontrivial solution $\phi$ to $\mathcal{H}$ for input $w \in$ $\mathcal{P C}_{W}$ with $\phi(0,0)=\xi \in \mathbb{R}^{n_{x}}$ if and only if $(\xi, w(0)) \in$ $D$ or

(VC): there exist $\epsilon>0$ and an absolutely continuous function $z:[0, \epsilon] \rightarrow \mathbb{R}^{n_{x}}$ such that $z(0)=\xi$, $\dot{z}(t) \in F(z(t), w(t))$ for almost all $t \in[0, \epsilon]$ and $(z(t), w(t)) \in C$ for all $t \in[0, \epsilon]$.

(iii) If condition (VC) holds for all $\xi \in \mathbb{R}^{n_{x}}$ and all $w \in$ $\mathcal{P C}_{W}$ with $(\xi, w(0)) \in C \backslash D$, then for all $\bar{w} \in \mathcal{P} \mathcal{C}_{W}$ every maximal solution $\phi \in \mathcal{S}_{\mathcal{H}}^{e}(\bar{w})$ satisfies exactly one of the following properties:

(a) $\phi$ is complete;

(b) $\phi$ is not complete and 'ends with flow': $\operatorname{dom} \phi$ is bounded and the interval $I^{J}:=\{t:(t, J) \in \operatorname{dom} \phi\}$ with $J=\sup _{j} \operatorname{dom} \phi$ is open to the right, and there does not exist an absolutely continuous function $z: \overline{I^{J}} \rightarrow$ $\mathbb{R}^{n_{x}}$ satisfying $\dot{z}(t) \in F(z(t), \bar{w}(t))$ for almost all $t \in I^{J}$ and $(z(t), \bar{w}(t)) \in C$ for all $t \in \operatorname{int} I^{J}$, and such that $z(t)=\phi(t, J)$ for all $t \in I^{J}$;

(c) $\phi$ is not complete and 'ends with a jump' or a 'discontinuity' of $\bar{w}: \operatorname{dom} \phi$ is bounded with $(T, J):=$ $\sup \operatorname{dom} \phi \in \operatorname{dom} \phi,(\phi(T, J), \bar{w}(T)) \notin C \cup D$.

As discussed before the theorem, note that we only have 
to verify (VC) over an interval $[0, \epsilon]$ of continuity of $w$.

As stated in (i) of Proposition 9, ae- and e- solutions coincide because, by closedness of $C$ and right-continuity of the considered input functions, solutions remain in $C$ during flow, except possibly at the end of a flow interval. Such an event necessarily corresponds to a discontinuity in the input $\bar{w}$. If the value of the state/input pair after the discontinuity of $\bar{w}$ is not in $C \cup D$, then the solution can neither flow nor jump and necessarily stops (case (c) in Proposition 9). See Example 7.

To illustrate the rationale behind our choice for càdlàg inputs, let us reconsider Example 5 demonstrating that imposing somewhat more regularity on the inputs leads to existence of nontrivial solutions as well as completeness of maximal solutions, while this was not the case for measurable inputs.

Example 6. Let us revisit Example 5 with the only difference that input signals are in $\mathcal{P} \mathcal{C}_{W}$ instead of in $\mathcal{L}_{W}$. With this restriction, we would like to show that existence of nontrivial solutions is now guaranteed for all initial states and all inputs in $\mathcal{P C}_{W}$ based on Proposition 9. If we compute $C \backslash D$ we obtain $C \backslash D=\left\{(\xi, w) \in \mathbb{R}^{2} \mid \xi+w>0\right\}$. As for any $w \in \mathcal{P C}_{W}$ there is an $\epsilon>0$ such that $w$ is continuous on $[0, \epsilon]$ and the solution to $\dot{x}=0$ is (locally absolutely) continuous too, we see that for every $\xi \in \mathbb{R}^{n_{x}}$ and $w \in \mathcal{P} \mathcal{C}_{W}$ with $(\xi, w(0)) \in C \backslash D$, (VC) holds due to the strict inequality in the expression for $C \backslash D$. It takes some positive time for the continuous function $t \mapsto(z(t), w(t))$ to leave $C$. Hence, the existence of nontrivial (ae-and $e$-)solutions is guaranteed and, moreover, in this case we can even show that each maximal solution is complete. Clearly, in contrast to Lebesgue measurable inputs in which these existence and completeness properties did not hold for the same hybrid system, they do hold when restricting inputs to $\mathcal{P} \mathcal{C}_{W}$.

Note that a similar conclusion can be drawn for system (10). For (10), existence of nontrivial solutions for all initial states and inputs in $\mathcal{P} \mathcal{C}_{W}$, as well as the $t$-completeness of maximal solutions is guaranteed, while this was not possible for all measurable inputs in $\mathcal{L}_{W}$ as observed in Example 4.

Remark 1. Extra care is required regarding item (c) of Proposition 9, if compared to the noninput case. In hybrid systems without inputs, it would be sufficient to prove that $G(D) \subset C \cup D$ to exclude (c). However, when dealing with discontinuous inputs as defined above, discontinuities in $w$ could also result in (c) occurring. See Example 7 for an illustration of this phenomenon.

Example 7. Consider system

$$
\begin{cases}\dot{x}=-x-w & x+w \leqslant 1 \\ x^{+}=-w & -2 \leqslant x+w \leqslant 2 .\end{cases}
$$

Take $W=\mathbb{R}$. It can be shown using Proposition 9 that for every initial state $\xi$ and every $w \in \mathcal{P C}_{W}$ with $(\xi, w(0)) \in$ $C \cup D$ a nontrivial solution exists. However, not all maximal solutions are complete due to case (c) mentioned in Proposition 9 occurring due to discontinuities in w. Indeed, take $\phi(0,0)=\xi=1$ and $w(t)=-1$ if $t \in[0,1)$ and $w(t)=2$ if $t \geqslant 1$. For this choice, a maximal (e-and ae)solution is given by $\phi(t, 0)=1, t \in[0,1]$ on the hybrid time domain $[0,1] \times\{0\}$. The discontinuity in $w$ at time 1 leads to $(\phi(1,0), w(1)) \notin C \cup D$. Note that jumps according to the jump map cannot lead to case (c) for this example.

\section{CONCLUSIONS}

Motivated by hybrid, networked, and event-triggered controllers for (physical) plants that are subject to disturbances, we studied hybrid systems with continuous-time measurable inputs that are given a priori. We presented two solution concepts, called ae- and e-solutions, and indicated subtle differences between them via various examples. Moreover, we provided tools to guarantee the existence of (nontrivial) ae- and e-solutions and completeness properties. Finally, by imposing additional "càdlàg " regularity on the continuoustime inputs, we obtained less stringent conditions for establishing basic existence and completeness results.

\section{REFERENCES}

[1] R. Goebel, R. G. Sanfelice, and A. R. Teel, Hybrid dynamical systems: modeling, stability, and robustness. Princeton University Press, 2012.

[2] C. Cai and A. R. Teel, "Characterizations of input-to-state stability for hybrid systems," Syst. \& Contr. Lett., vol. 58, no. 1, pp. 47-53, 2009.

[3] R. G. Sanfelice, Hybrid Feedback Control. Princ. Univ. Press, 2021.

[4] J. Cortes, "Discontinuous dynamical systems," IEEE Control Systems Magazine, vol. 28, no. 3, 2008.

[5] J. Lygeros, K. Johansson, S. Simic, J. Zhang, and S. Sastry, "Dynamical properties of hybrid automata," IEEE Trans. Aut. Control, vol. 48, no. 1, 2003.

[6] W. Heemels, M. Camlibel, A. Van der Schaft, and J. Schumacher, On the existence and uniqueness of solution trajectories to hybrid dynamical systems, ser. Nonlinear and Hybrid Systems in Automotive Control. Springer-Verlag, 2002, pp. 391-422.

[7] J. Chai and R. G. Sanfelice, "Forward Invariance of Sets for Hybrid Dynamical Systems (Part I)," IEEE Transactions on Automatic Control, vol. 64, no. 6, p. 2426-2441, Jun 2019.

[8] P. Bernard and R. G. Sanfelice, "Hybrid dynamical systems with hybrid inputs: Definition of solutions and applications to interconnections," Int. J. Robust and Nonlinear Control, vol. 30, no. 15, p. 5892-5916, 2020.

[9] S. van Loon, K. Gruntjens, M. Heertjes, N. van de Wouw, and W. Heemels, "Frequency-domain tools for stability analysis of reset control systems," Automatica, vol. 82, pp. 101-108, 2017.

[10] D. Nešić, L. Zaccarian, and A. R. Teel, "Stability properties of reset systems," Automatica, vol. 44, no. 8, pp. 2019-2026, 2008.

[11] C. Prieur, S. Tarbouriech, and L. Zaccarian, "Lyapunov-based hybrid loops for stability and performance of continuous-time control systems," Automatica, vol. 49, no. 2, pp. 577-584, 2013.

[12] C. Prieur and A. Teel, "Uniting local and global output feedback controllers," IEEE Trans. Aut. Contr., vol. 56, no. 7, pp. 1636-1649, 2011.

[13] W. Heemels, A. Teel, N. van de Wouw, and D. Nesic, "Networked control systems with communication constraints: Tradeoffs between transmission intervals, delays and performance," IEEE Trans. Aut. Contr., vol. 55, pp. 1781-1796, 2010.

[14] D. Nešić and A. Teel, "Input-to-state stability of networked control systems," Automatica, vol. 40, pp. 2121-2128, 2004.

[15] K. J. A. Scheres, R. Postoyan, and W. P. M. H. Heemels, "EventTriggered Control in Presence of Measurement Noise: A SpaceRegularization Approach," in IEEE Conf. Decision and Control, 2020, pp. 6234-6239.

[16] D. Borgers, V. Dolk, and W. Heemels, "Riccati-based design of event-triggered controllers for linear systems with delays," IEEE Transactions on Automatic Control, vol. 63, pp. 174-188, 2018.

[17] S. Mousavi, N. Noroozi, R. Geiselhart, M. Kögel, and R. Findeisen, "On integral input-to-state stability of event-triggered control systems," in IEEE Conf. Decision and Control, 2019, pp. 1674-1679. 